\title{
GENERALIZED GEODESIC DEVIATIONS: A LAGRANGEAN APPROACH
}

\author{
R. KERNER \\ L.P.T.L., Université Pierre et Marie Curie, CNRS URA 7600 \\ Tour 22, 4-ème étage, boîte 142, 4 place Jussieu, 75005 Paris, France \\ E-mail: rk@ccr.jussieu.fr
}

Dedicated to W. Tulczyjew on his 70th birthday

\begin{abstract}
The geodesic deviation equations, called also the Jacobi equations, describe only the first-order effects, linear in the small parameter characterizing the deviation from an original worldline. They can be easily generalized if we take into account the higher-order terms. Here we derive these higher-order equations not only directly, but also from the Taylor expansion of the variational principle itself. Then we show how these equations can be used in a novel approach to the two-body problem in General Relativity.
\end{abstract}

1. Introduction. A well-posed variational problem is consistent with respect to small deformations. This is the case of the curves of minimal length in Riemannian geometry, named geodesics. What we have in mind here is the following. If a variational principle is applied to a given Lagrangean depending on coordinate and velocity functions parametrized by a parameter $\tau$,

$$
\delta \int \mathcal{L}\left(x^{\mu}, \dot{x}^{\lambda}\right) d \tau=0, \quad\left(\text { with } \quad \dot{x}^{\mu}=d x^{\mu} / d \tau\right),
$$

it leads to the set of Euler-Lagrange equations

$$
\frac{d}{d \tau} \frac{\partial \mathcal{L}}{\partial \dot{x}^{\mu}}-\frac{\partial \mathcal{L}}{\partial x^{\mu}}=0
$$

Suppose now that the variables $x^{\mu}$, as well as the velocities $\dot{x}^{\mu}$ depend smoothly on a small deformation parameter $\epsilon$; then the Lagrangean function can be developed into a series in powers of $\epsilon$ under the integral, yielding corresponding series of independent variational principles (in what follows, $\mathcal{L}_{0}$ denotes just the initial Lagrangean function given in eq. (1), while the next integrands are obtained from the formal power expansion):

2000 Mathematics Subject Classification: 83-08, 83C25, 83C10.

The paper is in final form and no version of it will be published elsewhere. 


$$
\begin{aligned}
& \mathcal{L}\left(x^{\mu}(\tau, \epsilon), \dot{x}^{\lambda}(\tau, \epsilon)\right)= \mathcal{L}_{0}\left(x^{\mu}(\tau, 0), \dot{x}^{\lambda}(\tau, 0)\right)+\epsilon \mathcal{L}_{1}\left(x^{\mu}(\tau, \epsilon), \dot{x}^{\lambda}(\tau, \epsilon)\right) \\
&+\frac{\epsilon^{2}}{2 !} \mathcal{L}_{2}\left(x^{\mu}(\tau, 0), \dot{x}^{\lambda}(\tau, 0)\right)+\ldots \Rightarrow \\
& \delta \int \mathcal{L}_{0}\left(x^{\mu}, \dot{x}^{\lambda}\right) d \tau=0, \quad \delta \int \mathcal{L}_{1}\left(x^{\mu}, \dot{x}^{\lambda}\right) d \tau=0, \quad \delta \int \mathcal{L}_{2}\left(x^{\mu}, \dot{x}^{\lambda}\right) d \tau=0,
\end{aligned}
$$

and so on, leading to an infinite family of equations of motion which can be solved by iteration, starting from the zeroth order in $\epsilon$, then inserting the obtained zeroth-order solution into the equation resulting from the variational principle derived from the term proportional to $\epsilon$, and so forth.

In principle, one could start with the exact Euler-Lagrange equations derived from the single variational principle with the integrand not yet expanded in a series of powers of $\epsilon$, and then suppose that the solution we are looking for is a sum of infinite number of terms proportional to growing powers of $\epsilon$. If the problem is well posed, the two methods should lead asymptotically to the same result.

Of course, some singular cases are known, namely when the systems are subjected to constraints or symmetries; in general, after reduction to the irreducible phase space of essential variables, the above scheme may prove not to be satisfied, i.e. certain limits and developments do not lead to the same results before and after dimensional reduction. These problems have been investigated in a deep and sophisticated manner by Włodzimierz Tulczyjew and his collaborators during past three decades, and many of the new insights and results thus obtained have become part of the common wisdom in modern analytic mechanics and field theory (cf. [1], [2], [3])

The expansion of complicated non-linear problems into a series of approximate equations has been successfully applied in many domains of Physics, in related to the problem of motion. In General Relativity such expansions become the only reliable tool for the treatment of the two-body problem, because even the geodesic equation describing the motion of a test particle in a given field is non-linear, and so are the field equations that must be solved if one has to take into account the interaction between the particle and the field, with the field created by the particle itself. These methods have been initiated by the works of A. Einstein and L. Infeld ([4]), then by L. Infeld and J. Plebański ([5]), and later carried on by S. L. Bażański ([6], [7], [8]). In the eighties, powerful methods based on the post-Newtonian approximations have been elaborated by Ll. Bel, Th. Damour, N. Deruelle, G. Schaefer and L. Blanchet ([9], [10]), and their results are widely known.

Włodzimierz Tulczyjew in his early Warsaw years (1957-1965) contributed to the problem of spinning extended bodies in General Relativity, using an original approach based on the multipole expansion $([11],[12])$. I read this work while I was a graduate student, working partly under Tulczyjew's direction (while Andrzej Trautman was my principal advisor then). It is gratifying and pleasant to acknowledge now, after so many years, how the first genuine scientific work in statu nascendi that I read as a student has influenced my recent work on the two-body problem in General Relativity, including the spin effects. An old Jewish wisdom says that teaching a young man is like writing on a white page, while teaching an old man is like writing on the paper on which something 
is already written. Indeed it is true; and this is why the influence of Tulczyjew's work on me lasted so long.

In what follows, I shall describe recent results obtained in collaboration with A. Balakin, J.-W. van Holten and R. Colistete Jr. ([23], [25], [26]) based on the generalization and extension of the idea of multipole expansion applied here to geodesic deviations of arbitrary order. This technique enabled us to generate explicit approximate solutions by finding the geodesic deviations of higher orders and adding them up to a particularly simple exact solution which in this case is a test particle moving with constant velocity along a circle around the heavy mass described by Schwarzschild's metric.

2. Higher-order geodesic deviations. One of the most elegant ways to introduce the geodesic deviation equations and their higher-order generalizations makes use of the Lie derivatives and can be done in a totally coordinate-independent manner (see, for example, [31]). It is based on the hypothesis that a geodesic curve can be embedded into a many-parameter family ("congruence") of geodesics. The vector fields generated by motions along these new parameters are often called the Jacobi fields.

Given a one-parameter congruence of geodesics, one can define the tangent vector field $Z$ and the local Jacobi field $X$; then the Lie bracket of these fields vanishes (because the congruence spans a submanifold and therefore is integrable), so that $[X, Z]=0$. The geodesic equation is $\nabla_{Z} Z=0$. Applying the definition of Riemann tensor to the vectors $X, Y$ and $Z$, we get

$$
\left[\nabla_{X} \nabla_{Z}-\nabla_{Z} \nabla_{X}\right] Z-\nabla_{[X, Z]} Z=R(X, Z) Z
$$

and taking into account that $[X, Z]=0$ as well as the fact that $\nabla_{X} Z=\nabla_{Z} X$ and the anti-symmetry of $R(X, Z)$ in its two arguments, we get easily

$$
\nabla_{Z} \nabla_{X} Z=\nabla_{Z}^{2} X=R(Z, X) Z
$$

which coincides with the usual geodesic deviation equation when expressed in a local coordinate system.

One may continue in the same spirit and introduce two linearly independent Jacobi fields, $X$ and $Y$, both satisfying $[X, Z]=0=[Y, Z]$, to obtain the coordinate-independent form of Eq. (21), as follows. The two linearly independent Jacobi fields, $X$ and $Y$, satisfy $[X, Z]=0$ and $[Y, Z]=0$. By virtue of the Jacobi identity, we have $[[X, Y], Z]=0$, hence $[X, Y]$ is also a Jacobi field (i.e., it satisfies Eq. (6)). Applying the same formula to this field, we get

$$
\nabla_{Z}^{2}([X, Y])=R(Z,[X, Y]) Z
$$

Then, using the fact that $\nabla_{X} Y-\nabla_{Y} X=[X, Y]$, we can write the left hand side of the above equation as $\left.\nabla_{Z}^{2}\left(\nabla_{X} Y-\nabla_{Y} X\right]\right)$. This can be written explicitly as

$$
\nabla_{Z}^{2}\left(\nabla_{X} Y-\nabla_{Y} X\right)=R\left(Z, \nabla_{X} Y-\nabla_{Y} X\right) Z
$$

and furthermore, using the linearity property, as

$$
\nabla_{Z}^{2}\left(\nabla_{Y} X\right)-R\left(Z, \nabla_{Y} X\right) Z=\nabla_{Z}^{2}\left(\nabla_{X} Y\right)-R\left(Z, \nabla_{X} Y\right) Z .
$$

The left-hand side of the above equation coincides with the usual Jacobi equation applied to the field $\nabla_{Y} X$, whereas the right-hand side can be transformed using the definition of 
the Riemann tensor: the term $\nabla_{Z}^{2}\left(\nabla_{X} Y\right)$ gives

$$
\begin{aligned}
\nabla_{Z}^{2} Y & =\nabla_{Z}\left(\nabla_{Z} \nabla_{X} Y\right)=\nabla_{Z}\left(\nabla_{Z} \nabla_{X} Y-\nabla_{X} \nabla_{Z} Y\right)+\nabla_{Z}\left(\nabla_{X} \nabla_{Z} Y\right) \\
& =\nabla_{Z}(R(Z, X) Y)+\nabla_{Z}\left(\nabla_{X} \nabla_{Z} Y\right) \\
& =\left[\left(\nabla_{Z} R\right)(Z, X)\right] Y+R\left(Z, \nabla_{Z} X\right) Y+R(Z, X) \nabla_{Z} Y+\nabla_{Z}\left(\nabla_{X} \nabla_{Z} Y\right)
\end{aligned}
$$

(here we used the fact that $\nabla_{Z} Z=0$ ). Manipulating further in the same manner the commutators of covariant derivations, we arrive at

$$
\begin{gathered}
\nabla_{Z}^{2}\left(\nabla_{Y} X\right)-R\left(Z, \nabla_{Y} X\right) Z= \\
\nabla_{X} R(Z, Y) Z+\nabla_{Z} R(Z, X) Y+2 R(Z, Y) \nabla_{Z} X+2 R(Z, X) \nabla_{Z} Y .
\end{gathered}
$$

Although these coordinate-independent derivations are very elegant, the resulting expressions are not very useful for pratical computations. In practice, sooner or later one has to choose a coordinate system related to the observer, and translate all the calculations into the coordinate language. Then the same equations can be derived in a less abstract manner. Consider a (pseudo)-Riemannian manifold $V_{4}$ with the line element defined by the metric tensor $g_{\mu \nu}\left(x^{\lambda}\right)$,

$$
d s^{2}=g_{\mu \nu}\left(x^{\lambda}\right) d x^{\mu} d x^{\nu},
$$

a smooth curve $x^{\lambda}(s)$ parametrized with its own length parameter (or proper time) $s$ is a geodesic if its tangent vector $u^{\mu}=\left(d x^{\mu} / d s\right)$ satisfies the equation:

$$
u^{\lambda} \nabla_{\lambda} u^{\mu}=0 \quad \Leftrightarrow \quad \frac{D u^{\mu}}{D s}=\frac{d u^{\mu}}{d s}+\Gamma_{\lambda \rho}^{\mu} u^{\lambda} u^{\rho}=0 .
$$

where $\Gamma_{\rho \lambda}^{\mu}$ denote the Christoffel connection coefficients of the metric $g_{\mu \nu}$.

Suppose that a smooth congruence of geodesics is given, of which the geodesics are labeled by a continuous parameter $p$ : $x^{\mu}=x^{\mu}(s, p)$, such that the two independent tangent vector fields are defined by $u^{\mu}(s, p)=\left(\partial x^{\mu} / \partial s\right)$ and $n^{\mu}(s, p)=\left(\partial x^{\mu} / \partial p\right)$. It is easily established that the rates of change of the tangent vectors in the mutually defined directions are equal:

$$
n^{\lambda} \nabla_{\lambda} u^{\mu}=u^{\lambda} \nabla_{\lambda} n^{\mu} \Leftrightarrow \frac{D u^{\mu}}{D p}=\frac{D n^{\mu}}{D s}=\frac{\partial^{2} x^{\mu}}{\partial p \partial s}+\Gamma_{\lambda \rho}^{\mu} \frac{\partial x^{\lambda}}{\partial p} \frac{\partial x^{\rho}}{\partial s},
$$

by virtue of the symmetry of Christoffel symbols in their lower indices.

The Riemann tensor can be defined using covariant derivations along the two independent directions of the congruence:

$$
\left[u^{\lambda} \nabla_{\lambda}, n^{\rho} \nabla_{\rho}\right] Y^{\mu}=\left[\frac{D}{D s} \frac{D}{D p}-\frac{D}{D p} \frac{D}{D s}\right] Y^{\mu}=R_{\lambda \rho \sigma}^{\mu} \frac{\partial x^{\lambda}}{\partial s} \frac{\partial x^{\rho}}{\partial p} Y^{\sigma} .
$$

Replacing $Y^{\mu}$ by $u^{\mu}$ in the above formula, we get

$$
\left[u^{\lambda} \nabla_{\lambda}, n^{\rho} \nabla_{\rho}\right] u^{\mu}=R_{\lambda \rho \sigma}^{\mu} \frac{\partial x^{\lambda}}{\partial s} \frac{\partial x^{\rho}}{\partial p} u^{\sigma}=R_{\lambda \rho \sigma}^{\mu} u^{\lambda} u^{\sigma} n^{\rho} .
$$

By virtue of the geodesic equation (12) and Eq. (13), this can be written as

$$
u^{\lambda} \nabla_{\lambda}\left(n^{\rho} \nabla_{\rho} u^{\mu}\right)=\frac{D}{D s} \frac{D u^{\mu}}{D p}=\frac{D^{2} n^{\mu}}{D s^{2}}=R_{\lambda \rho \sigma}^{\mu} u^{\lambda} u^{\sigma} n^{\rho} .
$$


This first-order geodesic deviation equation is often called the Jacobi equation, and is manifestly covariant. In certain applications, Eq. (16) can be replaced by its more explicit, although non-manifestly covariant version:

$$
\frac{d^{2} n^{\mu}}{d s^{2}}+2 \Gamma_{\lambda \rho}^{\mu} u^{\lambda} \frac{d n^{\rho}}{d s}+\partial_{\sigma} \Gamma_{\lambda \rho}^{\mu} u^{\lambda} u^{\rho} n^{\sigma}=0
$$

In this form of the geodesic deviation equation one easily identifies the relativistic generalizations of the Coriolis-type and centrifugal-type inertial forces, represented respectively by the second and third terms of Eq. (17).

The geodesic deviation can be used to construct geodesics $x^{\mu}(s)$ close to a given reference geodesic $x_{0}^{\mu}(s)$, by an iterative procedure as follows. Let the two geodesics be members of a congruence as above, with $x^{\mu}(s)=x^{\mu}(s, p)$ and $x_{0}^{\mu}(s)=x^{\mu}\left(s, p_{0}\right)$. It follows by direct Taylor expansion, that

$$
\begin{aligned}
x^{\mu}(s, p) & =x^{\mu}\left(s, p_{0}\right)+\left.\left(p-p_{0}\right) \frac{\partial x^{\mu}}{\partial p}\right|_{\left(s, p_{0}\right)}+\left.\frac{1}{2}\left(p-p_{0}\right)^{2} \frac{\partial^{2} x^{\mu}}{\partial p^{2}}\right|_{\left(s, p_{0}\right)}+\ldots \\
& =x_{0}^{\mu}(s)+\delta x^{\mu}(s)+\frac{1}{2 !} \delta^{2} x^{\mu}(s)+\frac{1}{3 !} \delta^{3} x^{\mu}(s)+\ldots
\end{aligned}
$$

where the more compact notation $\delta^{n} x^{\mu}(s)$ describes the $n^{\text {th }}$-order geodesic deviation. Because $\left(p-p_{0}\right)$ is supposed to be a small quantity, for convenience we may denote it $\epsilon$. The first-order deviation is a vector, $\delta x^{\mu}(s)=\left(p-p_{0}\right) n_{0}^{\mu}(s)=\epsilon n_{0}^{\mu}(s)$. But the secondorder deviation is not a vector, and is given by

$$
\delta^{2} x^{\mu}(s)=\left(p-p_{0}\right)^{2}\left(b^{\mu}-\Gamma_{\lambda \nu}^{\mu} n^{\lambda} n^{\nu}\right)_{0}=\epsilon^{2}\left(b^{\mu}-\Gamma_{\lambda \nu}^{\mu} n^{\lambda} n^{\nu}\right)_{0}
$$

where the covariant second-order deviation vector $b^{\mu}$ is defined by

$$
b^{\mu}=\frac{D n^{\mu}}{D p}=\frac{\partial n^{\mu}}{\partial p}+\Gamma_{\lambda \nu}^{\mu} n^{\lambda} n^{\nu} .
$$

Straightforward covariant differentiation of Eq. (16), plus use of the Bianchi and Ricci identities for the Riemann tensor, implies that this second-order deviation vector $b^{\mu}(s)$ satisfies an inhomogeneous extension of the first-order geodesic deviation equation:

$$
\frac{D^{2} b^{\mu}}{D s^{2}}+R_{\rho \lambda \sigma}^{\mu} u^{\lambda} u^{\sigma} b^{\rho}=\left[\nabla_{\nu} R_{\lambda \rho \sigma}^{\mu}-\nabla_{\lambda} R_{\nu \sigma \rho}^{\mu}\right] u^{\lambda} u^{\sigma} n^{\rho} n^{\nu}+4 R_{\lambda \rho \sigma}^{\mu} u^{\lambda} n^{\rho}\left(\frac{D n^{\sigma}}{D s}\right) .
$$

A rigorous mathematical study of geodesic deviations up to the second-order, as well as geometric interpretation, but using different derivation, was presented in by Bażański in [6]. Also, a Hamilton-Jacobi formalism had been derived in [7] and applied to the problem of free falling particles in the Schwarzschild space-time [8]. Fine effects resulting from the analysis of geodesic deviations of test particles suspended in hollow spherical satellites have been discussed in [13].

Obviously the procedure can be extended to arbitrarily high order geodesic deviations $\delta^{n} x^{\mu}(s)$. This is of considerable practical importance, as it allows to construct a desired set of geodesics in the neighborhood of the reference $x_{0}^{\mu}(s)$, when the congruence of geodesics is not given a priori in closed form. Indeed, all that is needed is the set of deviation vectors $\left(n_{0}^{\mu}(s), b_{0}^{\mu}(s), \ldots\right)$ on the reference geodesic; obviously these vectors are completely specified as functions of $s$ by solving the geodesic deviation equations (16), 
(21) and their extensions to higher order, for given $x_{0}^{\mu}(s)$. As in the case of the firstorder deviation, it is sometimes convenient to write equation (21) in the equivalent but non-manifestly covariant form

$$
\begin{aligned}
& \frac{d^{2} b^{\mu}}{d s^{2}}+\partial_{\rho} \Gamma_{\lambda \sigma}^{\mu} u^{\lambda} u^{\sigma} b^{\rho}+2 \Gamma_{\lambda \sigma}^{\mu} u^{\lambda} \frac{d b^{\sigma}}{d s}=4\left(\partial_{\lambda} \Gamma_{\sigma \rho}^{\mu}+\Gamma_{\sigma \rho}^{\nu} \Gamma_{\lambda \nu}^{\mu}\right) \frac{d n^{\sigma}}{d s}\left(u^{\lambda} n^{\rho}-u^{\rho} n^{\lambda}\right) \\
& +\left(\Gamma_{\sigma \nu}^{\tau} \partial_{\tau} \Gamma_{\lambda \rho}^{\mu}+2 \Gamma_{\lambda \tau}^{\mu} \partial_{\rho} \Gamma_{\sigma \nu}^{\tau}-\partial_{\nu} \partial_{\sigma} \Gamma_{\lambda \rho}^{\mu}\right)\left(u^{\lambda} u^{\rho} n^{\sigma} n^{\nu}-u^{\sigma} u^{\nu} n^{\lambda} n^{\rho}\right) .
\end{aligned}
$$

The equation for the 3rd-order deviation can be found in our paper $([26])$.

The non-manifestly covariant geodesic deviation equations are often better adapted to deriving successive approximations for geodesics close to the initial one. Starting from a given geodesic $x^{\mu}(s)$ we can solve Eq. (17) and find the first-order deviation vector $n^{\mu}(s)$. Then, inserting $u^{\mu}(s)$ and $n^{\mu}(s)$, by now completely determined, into the system (22), we can solve and find the second-order deviation vector $b^{\mu}(s)$, and subsequently for the true second-order coordinate deviation $\delta^{2} x^{\mu}$, and so forth. As an example, below we describe non-circular motion, along with Kepler's laws (in an approximate version), together with the relativistic perihelion advance, starting from a circular orbit in Schwarzschild metric.

3. Lagrangean formulation of deviation equations including spin. The generalized geodesic deviation equations can be extended to the case of the particles carrying an electric charge and/or quasi-classical spin. In these cases particles do not move on geodesics, but on more general world lines [33]. For the case of charged particles in a combined electro-magnetic and gravitational field, the resulting world line deviation equation was derived in [25].

To this end, it is useful to consider the Lagrangean formulation of the geodesic deviation equations. We first observe that this equation is linear and homogeneous in $n^{\mu}$. It is therefore not very difficult to construct an action from which it can be derived. The Lagrangean of interest reads

$$
\mathcal{L}(n)=\frac{1}{2} g_{\mu \nu} \frac{D n^{\mu}}{D \tau} \frac{D n^{\nu}}{D \tau}+\frac{1}{2} R_{\mu \rho \nu \lambda} u^{\rho} u^{\lambda} n^{\mu} n^{\nu} .
$$

In this Lagrangean the metric, connection and curvature are those on the given reference geodesic $x_{0}^{\mu}(\tau)$, with $u^{\mu}(\tau)=\dot{x}_{0}^{\mu}$ representing the four-velocity. These quantities will be treated as background variables. Only the $n^{\mu}(\tau)$ should be considered as independent Lagrangean coordinates which are to be varied in the action. The action (23) can be derived independently by starting from the geodesic Lagrangean

$$
\mathcal{L}(x)=\frac{1}{2} g_{\mu \nu}(x) \frac{d x^{\mu}}{d \tau} \frac{d x^{\nu}}{d \tau},
$$

and expanding $x^{\mu}(\tau)$ near the given background geodesic solution in the form $x^{\mu}=$ $x_{0}^{\mu}+n^{\mu} \Delta \lambda$. The term independent of $\Delta \lambda$ does not contain $n^{\mu}$, and contributes, after integration, only a constant term to the action. Next all terms linear in $\Delta \lambda$ drop out of the result because $x_{0}$ is a solution of the geodesic equation. Finally, the terms quadratic in $\Delta \lambda$ reproduce the expression (23), up to a total proper-time derivative and terms which vanish because of the geodesic equation for $x_{0}^{\mu}(\tau)$. Thus the Lagrangean (23) represents the lowest-order non-trivial term in a systematic expansion of our action integral, as in 
the formula (3) of the Introduction:

$$
S[x]=m \int d \tau L\left(x_{0}\right)+m(\Delta \lambda)^{2} \int d \tau L(n)+\mathcal{O}\left[(\Delta \lambda)^{3}\right] .
$$

The higher-order approximations can also be derived in this way.

This derivation of the deviation equations can be also applied to the case of charged particles. One should start from the action

$$
S_{q}[x]=\int d \tau\left[\frac{m}{2} g_{\mu \nu}(x) \dot{x}^{\mu} \dot{x}^{\nu}+q A_{\mu}(x) \dot{x}^{\mu}\right],
$$

with the overdot the usual short-hand for proper-time derivatives. The world-lines generated by this action are solutions of the Einstein-Lorentz equation

$$
\frac{D^{2} x^{\mu}}{D \tau^{2}}=\frac{q}{m} F_{\nu}^{\mu} \frac{d x^{\nu}}{d \tau}
$$

Now given a solution $x_{0}^{\mu}(\tau)$ of this equation, and expanding the path in $S_{q}[x]$ as

$$
x^{\mu}(\tau)=x_{0}^{\mu}(\tau)+\Delta \lambda n^{\mu}(\tau),
$$

the action can be expanded to second order in $\Delta \lambda$ as

$$
\begin{aligned}
S_{q}[x]= & S_{q}\left[x_{0}\right]+\frac{(\Delta \lambda)^{2}}{2} \int d \tau\left[m\left(g_{\mu \nu} \frac{D n^{\mu}}{D \tau} \frac{D n^{\nu}}{D \tau}+R_{\mu \rho \nu \sigma} u^{\rho} u^{\sigma} n^{\mu} n^{\nu}\right)\right. \\
& \left.+q\left(F_{\mu \nu} n^{\mu} \frac{D n^{\nu}}{D \tau}+\nabla_{\mu} F_{\nu \rho} u^{\rho} n^{\mu} n^{\nu}\right)\right]+\mathcal{O}\left[(\Delta \lambda)^{3}\right] .
\end{aligned}
$$

To this order we then find that other solutions of the world-line equation (27), close to $x_{0}^{\mu}(\tau)$, are given by (28), with $n^{\mu}$ the solution of the world-line deviation equation $[24,25,23]$

$$
\frac{D^{2} n^{\mu}}{D \tau^{2}}=R_{\rho \nu \sigma}^{\mu} u^{\rho} u^{\sigma} n^{\nu}+\frac{q}{m} F_{\nu}^{\mu} \frac{D n^{\nu}}{D \tau}+\frac{q}{m} \nabla_{\rho} F_{\nu}^{\mu} u^{\nu} n^{\rho} .
$$

The alternative interpretation of $n^{\mu}$, as parametrizing the distance between two particles on neighboring world lines, holds in this case as well, provided the particles have the same charge-to-mass ratio $q / m$.

The same result can be obtained (30) from reduction of the geodesic equation and geodesic deviation equation in five-dimensional space-time, as particles with different $q / m$ ratio in four dimensions correspond to particles with different momentum in fivedimensional space-time, as shown in [23].

Similarly, pseudo-classical spinning particles can be described by the supersymmetric Lagrangean $[34,35]$

$$
\mathcal{L}_{\text {spin }}(x, \psi)=\frac{1}{2} g_{\mu \nu} \dot{x}^{\mu} \dot{x}^{\nu}+\frac{i}{2} \psi_{a} \frac{D \psi^{a}}{D \tau}
$$

with $\psi^{a}$ an anti-commuting tangent-space vector (note that the transition between basespace and tangent-space vectors is made as usual by the vierbein $e_{\mu}{ }^{a}$ and its inverse) such that the pseudo-classical spin is described by $S^{a b}=-i \psi^{a} \psi^{b}$. The corresponding equations of motion for spinning particles can be written as

$$
\frac{D^{2} x^{\mu}}{D \tau^{2}}=\frac{1}{2} S^{a b} R_{\nu a b}^{\mu} u^{\nu}, \quad \frac{D S^{a b}}{D \tau}=0 .
$$


Starting from a one-parameter congruence of solutions $\left(x^{\mu}(\tau ; \lambda), \psi^{a}(\tau ; \lambda)\right)$ we define the deviation vectors

$$
n^{\mu}=\frac{\partial x^{\mu}}{\partial \lambda}, \quad \xi^{a}=\frac{D \psi^{a}}{D \lambda}=\frac{\partial \psi^{a}}{\partial \lambda}-n^{\mu} \omega_{\mu b}^{a} \psi^{b},
$$

where $\omega_{\mu b}^{a}$ is the spin connection. The covariant change in the spin-tensor is then

$$
J^{a b}=\frac{D S^{a b}}{D \lambda}=-i\left[\psi^{a} \xi^{b}+\xi^{a} \psi^{b}\right]
$$

These vectors satisfy the world-line deviation equations

$$
\begin{aligned}
\frac{D^{2} n^{\mu}}{D \tau^{2}} & =R_{\sigma \nu \rho}^{\mu} u^{\sigma} u^{\rho} n^{\nu}+\frac{1}{2} S^{a b} R_{\nu a b}^{\mu} \frac{D n^{\nu}}{D \tau}+\frac{1}{2}\left[S^{a b} \nabla_{\rho} R_{\nu a b}^{\mu} u^{\nu} n^{\rho}+J^{a b} R_{\nu a b}^{\mu} u^{\nu}\right], \\
\frac{D J^{a b}}{D \tau} & =\left[S, R_{\mu \nu}\right]^{a b} u^{\mu} n^{\nu} .
\end{aligned}
$$

They define the stationary points of the quadratic deviation action

$$
\begin{aligned}
\mathcal{L}_{\text {spin }}(n, \xi)= & \frac{1}{2} g_{\mu \nu} \frac{D n^{\mu}}{D \tau} \frac{D n^{\nu}}{D \tau}+\frac{i}{2} \xi_{a} \frac{D \xi^{a}}{D \tau}+\frac{1}{2} R_{\mu \rho \nu \sigma} u^{\rho} u^{\sigma} n^{\mu} n^{\nu} \\
& -\frac{i}{4} \psi^{a} \psi^{b}\left[R_{\mu \nu a b} n^{\mu} \frac{D n^{\nu}}{D \tau}+\nabla_{\mu} R_{\nu \sigma a b} u^{\sigma} n^{\mu} n^{\nu}\right]-i R_{\mu \nu a b} n^{\mu} u^{\nu} \xi^{a} \psi^{b}
\end{aligned}
$$

This set of coupled equations is of interest for the analysis of fine gyroscopic effects, namely in the field of Kerr's metric.

4. Application to the Kepler problem in General Relativity. Although for orbital motion in a Schwarzschild background we have at our disposal the exact solutions in terms of quadratures (with integrals of elliptic or Jacobi type), our approach is particularly well-suited for numerical computations, because in appropriate (Gaussian) coordinates the geodesic curves can display a very simple parametric form, and all the components of the 4 -velocity and other quantities reduce to constants when restricted to that geodesic.

In this case equation (17) reduces to a linear system with constant coefficients, which after diagonalization becomes a collection of harmonic oscillators, and all that remains is to find the characteristic frequencies. In the next step, we get a collection of harmonic oscillators excited by external periodic forces represented by the right-hand side of (22), which can also be solved very easily, and so forth.

In the third order, the presence of resonances giving rise to secular terms could lead to instability of the orbit we started with; but this phenomenon can be dealt with by Poincaré's method [14], according to which such terms can be eliminated if we admit that the frequency of the resulting solution is also slightly modified, and can be expanded in a formal series of successive powers of the initial (small) deformation parameter.

At the end, the deviation becomes a series of powers of a small parameter containing linear combination of characteristic frequencies appearing on the right-hand side, which are entire multiples of the basic frequency, also slightly deformed. This description of planetary motion as a superposition of different harmonic motions has been first introduced by Ptolemaeus in the II century [15]. We shall now analyse the simplest case of circular orbits in Schwarzschild geometry. 
Consider the geodesic deviation equation starting with a circular orbit in the field of a spherically-symmetric massive body. The circular orbits and their stability have been analyzed and studied in several papers [21, 22] and books, e.g. the monograph by Chandrasekhar [16]. The gravitational field is described by the line-element (in natural coordinates with $c=1$ and $G=1$ )

$$
g_{\mu \nu} d x^{\mu} d x^{\nu}=-d s^{2}=-B(r) d t^{2}+\frac{1}{B(r)} d r^{2}+r^{2}\left(d \theta^{2}+\sin ^{2} \theta d \phi^{2}\right),
$$

with $B(r)=1-2 M / r$.

Let us recall the essential features of the solution of the geodesic equations for a test particle of mass $m<<M$. As the spherical symmetry guarantees conservation of angular momentum, the particle orbits are always confined to an equatorial plane $\theta=\pi / 2$. The constant angular momentum $J$ is then directed along the $z$-axis; let us denote its magnitude per unit of mass by $\ell=J / m$. In addition, as the metric is static outside the horizon $r_{+}=2 M$, it allows a time-like Killing vector which guarantees the existence of a conserved world-line energy (per unit of mass $m$ ) $\varepsilon$, so that we have:

$$
\frac{d \phi}{d s}=\frac{\ell}{r^{2}} \quad \text { and } \quad \frac{d t}{d s}=\frac{\varepsilon}{1-\frac{2 M}{r}} .
$$

Finally, the equation for the radial coordinate $r$ can be integrated owing to the conservation of the world-line Hamiltonian, i.e. the conservation of the absolute four-velocity:

$$
\left(\frac{d r}{d s}\right)^{2}=\varepsilon^{2}-\left(1-\frac{2 M}{r}\right)\left(1+\frac{\ell^{2}}{r^{2}}\right) .
$$

From this we derive a simplified expression for the radial acceleration:

$$
\frac{d^{2} r}{d s^{2}}=-\frac{M}{r^{2}}+\left(\frac{\ell^{2}}{r^{3}}\right)\left(1-\frac{3 M}{r}\right) .
$$

The equation (39) can in principle be integrated directly; indeed, the orbital function $r(\phi)$ is given by an elliptic integral $[17,18]$. However, to get directly an approximate parametric solution to the equations of motion one can also study perturbations of special simple orbits. In the following we study the problem for bound orbits by considering the first and second-order geodesic deviation equations for the special case of world lines close to circular orbits.

Observe that for circular orbits $r=R=$ constant, the expressions for $d r / d s$, Eq. (39) and $d^{2} r / d s^{2}$, Eq. (40) must both vanish at all times. This produces two relations between the three dynamical quantities $(R, \varepsilon, \ell)$, showing that the circular orbits are characterized completely by specifying either the radial coordinate, or the energy, or the angular momentum of the planet. In particular, the equation for null radial velocity gives

$$
\varepsilon^{2}=\left(1-\frac{2 M}{R}\right)\left(1+\frac{\ell^{2}}{R^{2}}\right) .
$$

Then condition (40) gives the well-known result $M R^{2}-\ell^{2}(R-3 M)=0$ leading to the requirement $R \geq 6 M$ for stable circular orbits to exist.

With this in mind, and the explicit formulae for the Christoffel coefficients of Schwarzschild's metric, we can establish now the four differential equations that must be satisfied 
by the geodesic deviation 4 -vector $n^{\mu}(s)$ close to a circular orbit. We recall that on the circular orbit of radius $R$ (which is a geodesic in the background Schwarzschild metric) we have:

$$
u^{t}=\frac{d t}{d s}=\frac{\varepsilon}{\left(1-\frac{2 M}{R}\right)}, \quad u^{r}=\frac{d r}{d s}=0, \quad u^{\phi}=\frac{d \varphi}{d s}=\omega_{0}=\frac{\ell}{R^{2}}, \quad u^{\theta}=\frac{d \theta}{d s}=0,
$$

because $r=R=$ const., $\theta=\pi / 2=$ const., so that $\sin \theta=1$ and $\cos \theta=0$.

Using the explicit form of the first-order deviation equation 16 , we get without effort the first three equations, for the components $n^{\theta}, n^{\phi}$ and $n^{t}$ :

$$
\begin{gathered}
\frac{d^{2} n^{\theta}}{d s^{2}}=-\left(u^{\phi}\right)^{2} n^{\theta}=-\frac{\ell^{2}}{R^{4}} n^{\theta} \\
\frac{d^{2} n^{\phi}}{d s^{2}}=-\frac{2 \ell}{R^{3}} \frac{d n^{r}}{d s}, \quad \frac{d^{2} n^{t}}{d s^{2}}=-\frac{2 M \varepsilon}{R^{2}\left(1-\frac{2 M}{R}\right)^{2}} \frac{d n^{r}}{d s} .
\end{gathered}
$$

The deviation $n^{\theta}$ is independent of the remaining three variables $n^{t}, n^{r}$ and $n^{\varphi}$. The harmonic oscillator equation (43) for $n^{\theta}$ displays the frequency which is equal to the frequency of the circular motion of the planet itself:

$$
n^{\theta}(s)=n_{0}^{\theta} \cos \left(\omega_{0} s+\gamma\right)=n_{0}^{\theta} \cos \left(\frac{\ell}{R^{2}} s+\gamma\right) .
$$

This can be interpreted as the result of a change of the coordinate system, with a new $z$-axis slightly inclined with respect to the original one, so that the plane of the orbit does not coincide with the plane $z=0$. In this case the deviation from the plane will be described by the above solution, i.e. a trigonometric function with the period equal to the period of the planetary motion. This allows us to eliminate the variable $n^{\theta}$ by choosing $n^{\theta}=0$.

It takes a little more time to establish the equation for $n^{r}$, using Eq. (17):

$$
\frac{d^{2} n^{r}}{d s^{2}}+2 \Gamma_{\lambda \rho}^{r} u^{\lambda} \frac{d n^{\rho}}{d s}+\partial_{\sigma} \Gamma_{\lambda \rho}^{r} u^{\lambda} u^{\rho} n^{\sigma}=0 .
$$

Taking into account that only the components $u^{t}$ and $u^{\phi}$ of the four-velocity on the circular orbit are different from zero, and recalling that we have chosen to set $n^{\theta}=0$, too, the only non-vanishing terms in the above equation are:

$$
\frac{d^{2} n^{r}}{d s^{2}}+2 \Gamma_{t t}^{r} u^{t} \frac{d n^{t}}{d s}+2 \Gamma_{\phi \phi}^{r} u^{\phi} \frac{d n^{\phi}}{d s}+\partial_{r} \Gamma_{t t}^{r} u^{t} u^{t} n^{r}+\partial_{r} \Gamma_{\phi \phi}^{r} u^{\phi} u^{\phi} n^{r}=0 .
$$

Using the identities and the definitions (42), we get

$$
\frac{d^{2} n^{r}}{d s^{2}}-\frac{3 \ell^{2}}{R^{4}}\left(1-\frac{2 M}{R}\right) n^{r}+\frac{2 M \varepsilon}{R^{2}} \frac{d n^{t}}{d s}-\frac{2 \ell}{R}\left(1-\frac{2 M}{R}\right) \frac{d n^{\varphi}}{d s}=0 .
$$

The system of three remaining equations can be expressed in a matrix form:

$$
\left(\begin{array}{ccc}
\frac{d^{2}}{d s^{2}} & \frac{2 M \varepsilon}{R^{2}\left(1-\frac{2 M}{R}\right)^{2}} \frac{d}{d s} & 0 \\
\frac{2 M \varepsilon}{R^{2}} \frac{d}{d s} & \frac{d^{2}}{d s^{2}}-\frac{3 \ell^{2}}{R^{4}}\left(1-\frac{2 M}{R}\right) & -\frac{2 \ell}{R}\left(1-\frac{2 M}{R}\right) \frac{d}{d s} \\
0 & \frac{2 \ell}{R^{3}} \frac{d}{d s} & \frac{d^{2}}{d s^{2}}
\end{array}\right)\left(\begin{array}{c}
n^{t} \\
n^{r} \\
n^{\varphi}
\end{array}\right)=\left(\begin{array}{l}
0 \\
0 \\
0
\end{array}\right)
$$


With the help of the identity (41) the characteristic equation of the above matrix reduces to

$$
\lambda^{4}\left[\lambda^{2}+\frac{\ell^{2}}{R^{4}}\left(1-\frac{6 M}{R}\right)\right]=0,
$$

so that the characteristic circular frequency is

$$
\omega=\frac{\ell}{R^{2}} \sqrt{1-\frac{6 M}{R}}=\omega_{0} \sqrt{1-\frac{6 M}{R}} .
$$

It is obvious that the general solution contains oscillating terms $\cos (\omega s)$. The presence of first and second-order derivatives in the matrix operator (49) means that the general solution might contain also constants and terms linear in $s$; however, these turn out to describe trivial solutions resulting from changing $R$ into $R+\delta R$, and adapting the constant values of $u^{t}$ and of $u^{\varphi}$.

Therefore, taking into account only the non-trivial, oscillatory part of the solution, and choosing the initial phase to have (with $n_{0}^{r}>0$ ), we get

$$
n^{r}(s)=-n_{0}^{r} \cos (\omega s) .
$$

What remains to be done is to compare this frequency with the fundamental circular frequency $\omega_{0}=\ell / R^{2}$ of the unperturbed circular orbital motion.

But this discrepancy between the two circular frequencies $\omega$ and $\omega_{0}$ is exactly what produces the perihelion advance, and its value coincides with the value obtained in the usual way in the limit of quasi-circular orbits, i.e. when $e^{2} \rightarrow 0$ : we get both the correct value and the correct sign.

Here is the complete solution for the first-order deviation vector $n^{\mu}(s)$ :

$$
n^{\theta}=0, n^{r}(s)=-n_{0}^{r} \cos (\omega s), n^{\varphi}=n_{0}^{\varphi} \sin (\omega s), n^{t}=n_{0}^{t} \sin (\omega s) .
$$

The only independent amplitude is given by $n_{0}^{r}$, because we have

$$
\begin{aligned}
& n_{0}^{t}=\frac{2 M \varepsilon}{R^{2}\left(1-\frac{2 M}{R}\right)^{2} \omega} n_{0}^{r}=\frac{2 \sqrt{M}}{\sqrt{R}\left(1-\frac{2 M}{R}\right) \sqrt{1-\frac{6 M}{R}}} n_{0}^{r}, \\
& n_{0}^{\varphi}=\frac{2 \ell}{R^{3} \omega} n_{0}^{r}=\frac{2 \omega_{0}}{R \omega} n_{0}^{r}=\frac{2}{R \sqrt{1-\frac{6 M}{R}}} n_{0}^{r} .
\end{aligned}
$$

So the trajectory and the law of motion are given by

$$
\begin{aligned}
& r=R-n_{0}^{r} \cos (\omega s), \\
& \varphi=\omega_{0} s+n_{0}^{\varphi} \sin (\omega s)=\frac{\sqrt{M}}{R^{3 / 2} \sqrt{1-\frac{3 M}{R}}} s+n_{0}^{\varphi} \sin (\omega s), \\
& t=\frac{\varepsilon}{\left(1-\frac{2 M}{R}\right)} s+n_{0}^{t} \sin (\omega s)=\frac{1}{\sqrt{1-\frac{3 M}{R}}} s+n_{0}^{t} \sin (\omega s),
\end{aligned}
$$

where the phase in the argument of the cosine function was chosen so that $s=0$ corresponds to the perihelion, and $s=\pi / \omega$ to the aphelion. It is important to note once again that the coefficient $n_{0}^{r}$, which also fixes the values of the two remaining amplitudes, $n_{0}^{t}$ 
and $n_{0}^{\varphi}$, defines the size of the actual deviation, so that the ratio $n_{0}^{r} / R$ becomes the dimensionless infinitesimal parameter controlling the approximation series with consecutive terms proportional to the consecutive powers of $n_{0}^{r} / R$.

What we see here is the approximation to an elliptic orbital movement as described by the presence of an epicycle like in the Ptolemean system [15], except that the Sun is placed in the center instead of the Earth. Indeed, the development into power series with respect to the eccentricity $e$ considered as a small parameter, and leaving only the linear term coincides with the Kepler result [19],

$$
r(t)=\frac{a\left(1-e^{2}\right)}{1+e \cos \left(\omega_{0} t\right)} \simeq a\left[1-e \cos \left(\omega_{0} t\right)\right],
$$

which looks almost as our formula (56) if we identify the eccentricity $e$ with $n_{0}^{r} / R$ and the greater half-axis $a$ with $R$; but there is also the additional difference, that the circular frequency of the epicycle is now slightly lower than the circular frequency of the unperturbed circular motion. But if the circular frequency is lower, the period is slightly longer; and as one must have $\Delta \varphi / 2 \pi=\Delta T / T_{0} \simeq-\Delta \omega / \omega_{0}$, we obtain an approximation (linear in the eccentricity) for the perihelion advance after one revolution which may be developed into a Taylor series in $M / R$ :

$$
\Delta \varphi=\frac{6 \pi M}{R}+\frac{27 \pi M^{2}}{R^{2}}+\frac{135 \pi M^{3}}{R^{3}}+\ldots
$$

It is obvious that at this order of approximation we could not keep track of the factor $\left(1-e^{2}\right)^{-1}$, containing the eccentricity (here replaced by the ratio $n_{0}^{r} / R$ ) only through its square. In contrast, we obtain without effort the coefficients in front of terms quadratic or cubic in $M / R$. This shows that our method can be of interest when one has to consider the low-eccentricity orbits in the vicinity of very massive and compact bodies, having a non-negligible ratio $M / R$.

In order to include this effect, at least in its approximate form as the factor $\left(1+e^{2}\right)$, we must go beyond the first-order deviation equations and investigate the solutions of the equations describing the quadratic effects (22).

5. The second and third-order geodesic deviations. After inserting the complete solution for the first-order deviation vector (53)-(55) into the system (21) and a tedious calculation, we find the following set of linear equations satisfied by the second-order deviation vector $b^{\mu}(s)$ :

$$
\left(\begin{array}{ccc}
\frac{d^{2}}{d s^{2}} & \frac{2 M \varepsilon}{R^{2}\left(1-\frac{2 M}{R}\right)^{2}} \frac{d}{d s} & 0 \\
\frac{2 M \varepsilon}{R^{2}} \frac{d}{d s} & \frac{d^{2}}{d s^{2}}-\frac{3 \ell^{2}}{R^{4}}\left(1-\frac{2 M}{R}\right) & -\frac{2 \ell}{R}\left(1-\frac{2 M}{R}\right) \frac{d}{d s} \\
0 & \frac{2 \ell}{R^{3}} \frac{d}{d s} & \frac{d^{2}}{d s^{2}}
\end{array}\right)\left(\begin{array}{c}
b^{t} \\
b^{r} \\
b^{\varphi}
\end{array}\right)=\left(n_{0}^{r}\right)^{2}\left(\begin{array}{c}
C^{t} \\
C^{r} \\
C^{\varphi}
\end{array}\right)
$$

where we have put into evidence the common factor $\left(n_{0}^{r}\right)^{2}$, which shows the explicit quadratic dependence of the second-order deviation vector $b^{\mu}$ on the first-order deviation amplitude $n_{0}^{r}$. The constants $C^{t}, C^{r}$ and $C^{\varphi}$ are expressions depending on $M, R, \omega_{0}, \omega$, $\varepsilon, \sin (2 \omega s)$ and $\cos (2 \omega s)$. We shall not display them here; the complete calculus can be found in the article [26]. 
The solution of the above matrix equation for $b^{\mu}(s)$ has the same characteristic eigenvalues as the matrix (49) for $n^{\mu}(s)$, and the general solution containing oscillating terms with angular frequency $\omega$ is of no interest because it is already accounted for by $n^{\mu}(s)$. But the particular solution includes constants, the terms linear in $s$, and the terms oscillating with angular frequency $2 \omega$ :

$$
\begin{aligned}
b^{t} & =\frac{\left(n_{0}^{r}\right)^{2} M \varepsilon}{R^{3}\left(1-\frac{6 M}{R}\right)\left(1-\frac{2 M}{R}\right)^{2}}\left[-\frac{3\left(2-\frac{5 M}{R}+\frac{18 M^{2}}{R^{2}}\right)}{1-\frac{6 M}{R}} s+\frac{2-\frac{13 M}{R}}{\omega} \sin (2 \omega s)\right], \\
b^{r} & =\frac{\left(n_{0}^{r}\right)^{2}}{2 R\left(1-\frac{6 M}{R}\right)}\left[\frac{3\left(2-\frac{5 M}{R}+\frac{18 M^{2}}{R^{2}}\right)}{1-\frac{6 M}{R}}+\left(2+\frac{5 M}{R}\right) \cos (2 \omega s)\right], \\
b^{\varphi} & =\frac{\left(n_{0}^{r}\right)^{2} \omega_{0}}{R^{2}\left(1-\frac{6 M}{R}\right)}\left[-\frac{3\left(2-\frac{5 M}{R}+\frac{18 M^{2}}{R^{2}}\right)}{1-\frac{6 M}{R}} s+\frac{1-\frac{8 M}{R}}{2 \omega} \sin (2 \omega s)\right] .
\end{aligned}
$$

Now we need to calculate the quantities $\frac{1}{2} \delta^{2} x^{\mu}$ and add them to the coordinates of the original geodesic curve in order to obtain the geodesic curve $x^{\mu}$ with second-order geodesic deviation. They have the same form as the covariant deviations above, and can be found in our paper $([26])$.

The fact that the second-order deviation vector $b^{\mu}$ turns with angular frequency $2 \omega$ enables us to get a better approximation of the elliptic shape of the resulting orbit. The trajectory described by $x^{\mu}$ including second-order deviations is not an ellipse, but we can match the perihelion and aphelion distances to see that $R \neq a$ and $e \neq n_{0}^{r} / R$ when second-order deviation is used. The perihelion and aphelion distances of the Keplerian, i.e., elliptical orbit are $a(1-e)$ and $a(1+e)$. For $x^{\mu}$, the perihelion is obtained when $\omega s=2 k \pi$ and the aphelion when $\omega s=(1+2 k) \pi$, where $k \in \mathbf{Z}$. Matching the radius for perihelion and aphelion, we obtain the semimajor axis $a$ and the eccentricity $e$ of an ellipse that has the same perihelion and aphelion distances of the orbit described by $x^{\mu}$ :

$$
\begin{aligned}
& a=R+\frac{\left(n_{0}^{r}\right)^{2}}{12 R}\left[-1+\frac{3}{1-\frac{2 M}{R}}+\frac{7}{1-\frac{6 M}{R}}+\frac{15}{\left(1-\frac{6 M}{R}\right)^{2}}\right], \\
& e=\frac{n_{0}^{r}\left(1-\frac{2 M}{R}\right)\left(1-\frac{6 M}{R}\right)^{2}}{R\left(1-\frac{2 M}{R}\right)\left(1-\frac{6 M}{R}\right)^{2}+\frac{\left(n_{0}^{r}\right)^{2}}{R}\left[2-\frac{9 M}{R}+\frac{11 M^{2}}{R^{2}}+\frac{6 M^{3}}{R^{3}}\right]}=\frac{n_{0}^{r}}{R}+\mathcal{O}\left(\frac{\left(n_{0}^{r}\right)^{3}}{R^{3}}\right) .
\end{aligned}
$$

In the limit case of $M / R \rightarrow 0$, there is no perihelion advance and $a=R\left[1+2\left(n_{0}^{r} / R\right)^{2}\right]$ and $e=n_{0}^{r} / R$, so the second-order deviation increases the semimajor axis $a$ of a matching ellipse compared to the first-order deviation, when $a=R$ and $e=n_{0}^{r} / R$.

Now, from $\varphi(s)$ it is possible to write $s(\varphi)$ by means of successive approximations, starting with $\omega s=\varphi \sqrt{1-6 M / R}$. Finally, $s$ can be replaced in $r(s)$ and we obtain $r(\varphi)$ up to the second order in $n_{0}^{r} / R$ :

$$
\begin{aligned}
\frac{r}{R}= & -\frac{n_{0}^{r}}{R} \cos \left(\frac{\omega}{\omega_{0}} \varphi\right)+\left(\frac{n_{0}^{r}}{R}\right)^{2}\left[\frac{3-\frac{5 M}{R}-\frac{30 M^{2}}{R^{2}}+\frac{72 M^{3}}{R^{3}}}{2\left(1-\frac{2 M}{R}\right)\left(1-\frac{6 M}{R}\right)^{2}}\right. \\
& \left.+\frac{\left(1-\frac{5 M}{R}\right)}{2\left(1-\frac{6 M}{R}\right)} \cos \left(\frac{2 \omega}{\omega_{0}} \varphi\right)\right]+\ldots
\end{aligned}
$$


In the limit $M / R \rightarrow 0$, the exact equation of an ellipse is obtained up to the second order in $e$, where $e=n_{0}^{r} / R$ and $r_{0}=\left(1+e^{2}\right) R$ :

$$
r=\frac{r_{0}}{1+e \cos \varphi}=\frac{\left(1+e^{2}\right) R}{1+e \cos \varphi}=R\left[1-e \cos \varphi+e^{2}\left(\frac{3}{2}+\frac{1}{2} \cos 2 \varphi\right)+\ldots\right] .
$$

Comparing with the ellipse equation (59), we have $r_{0}=a\left(1-e^{2}\right)$, so that $a=$ $R\left(1+e^{2}\right) /\left(1-e^{2}\right) \simeq R\left(1+2 e^{2}\right)$ which agrees with the analysis of Eqs. (65)-(66).

With the third-order approximation we are facing a new problem, arising from the presence of resonance terms on the right-hand side. It is easy to see that after reducing the expressions on the right-hand side of the equation for the third-order deviation, which contain the terms of the form $\cos ^{3} \omega s, \sin \omega s \cos ^{2} \omega s$ and the like, we shall get not only the terms containing $\sin 3 \omega s$, and $\cos 3 \omega s$, which do not create any particular problem, but also the resonance terms containing the functions $\sin \omega s$ and $\cos \omega s$, whose circular frequency is the same as the eigenvalue of the matrix-operator acting on the left-hand side.

The equation for the covariant third-order deviation $h^{\mu}$ can be written in matrix form, with principal part linear in the third-order deviation $h^{\mu}$, represented by exactly the same differential operator (which we denote here symbolically by $\mathcal{M}$ ) as in the lowerorder deviation equations. The right-hand side is separated into two parts, one oscillating with frequency $\omega$, and another with frequency $3 \omega$ :

$$
[\mathcal{M}]\left(\begin{array}{c}
h^{t} \\
h^{r} \\
h^{\varphi}
\end{array}\right)=\left(n_{0}^{r}\right)^{3}\left(\begin{array}{c}
B^{t} \sin (\omega s)+C^{t} \sin (3 \omega s)+s D^{t} \cos (\omega s) \\
B^{r} \cos (\omega s)+C^{r} \cos (3 \omega s)+s D^{r} \sin (\omega s) \\
B^{\varphi} \sin (\omega s)+C^{\varphi} \sin (3 \omega s)+s D^{\varphi} \cos (\omega s)
\end{array}\right)
$$

where the coefficients $B^{k}, C^{k}$ and $D^{k}, k=t, r, \varphi$ are complicated functions of $M / R$.

The proper frequency of the matrix operator acting on the left-hand side is equal to $\omega$; the terms containing the triple frequency $3 \omega$ will give rise to the unique non-singular solution of the same frequency, but the resonance terms of the basic frequency on the right-hand side will give rise to secular terms, proportional to $s$, in contradiction with the bounded character of the deviation supposed from the beginning. The term proportional to $s$ on the right-hand side is eliminated in the differential equation for $h^{r}$ when $d h^{\varphi} / d s$ and $d h^{t} / d s$ are replaced by their values.

Poincaré [14] was the first to solve this apparent contradiction by taking into account possible perturbation of the basic frequency itself, which amounts to the replacement of $\omega$ by an infinite series in powers of the infinitesimal parameter, which in our case is the eccentricity $e=n_{0}^{r} / R$ :

$$
\omega \rightarrow \omega+e \omega_{1}+e^{2} \omega_{2}+e^{3} \omega_{3}+\ldots,
$$

Then, developing both sides into a series of powers of the parameter $e$, we can not only recover the former differential equations for the vectors $n^{\mu}, b^{\mu}, h^{\mu}$, but get also some algebraic relations defining the corrections $\omega_{1}, \omega_{2}, \omega_{3}$, etc.

The equations resulting from the requirement that all resonant terms on the righthand side be canceled by similar terms on the left-hand side are rather complicated. We do not attempt to solve them here. However, one easily observes that the absence of resonant terms in the second-order deviation equations forces $\omega_{1}$ to vanish, while the next term $\omega_{2}$ is different from 0 . 
This technique can be obviously extended to the next orders of approximation; it is clear that in the present case, the correction $\omega_{3}$ will be also equal to 0 , and the next non-vanishing secular terms will appear at the fifth-order approximation, as products of the type $\cos ^{5} \omega s, \sin ^{3} \omega s \cos ^{2} \omega s$, etc., produce resonant terms again, which will enable us to find the correction $\omega_{4}$, and so on, so that the resulting series representing the frequency $\omega$ contains only even powers of the small parameter $n_{0}^{r} / R$.

\section{References}

[1] J. Kijowski and W. M. Tulczyjew, A Symplectic Framework for Field Theories, Springer, 1979.

[2] J. Śniatycki, Geometric Quantization and Quantum Mechanics, Springer, 1980.

[3] W. M. Tulczyjew, Acta Physica Polonica B 8 (1977), 431.

[4] A. Einstein, Ann. Physik 49 (1916), 769.

[5] L. Infeld and J. Plebański, Motion and Relativity, PWN - Pergamon Press, 1961.

[6] S. L. Bażański, Ann. Inst. H. Poincaré A 27 (1977), 115.

[7] S. L. Bażański, Ann. Inst. H. Poincaré A 27 (1977), 145; J. Math. Phys. 30 (1989), 1018.

[8] S. L. Bażański and P. Jaranowski, J. Math. Phys. 30 (1989), 1794.

[9] T. Damour and N. Deruelle, Ann. Inst. H. Poincaré (Phys. Théor.) 43 (1985), 107; Ann. Inst. H. Poincaré (Phys. Théor.) 44 (1986), 263.

[10] L. Blanchet and G. Schäfer, Class. Quantum Grav. 10 (1993), 2699, and references therein.

[11] W. M. Tulczyjew, Bull. Acad. Polon. Sci. Cl III 6 (1958), 645.

[12] W. M. Tulczyjew, Acta Phys. Polon. 18 (1959), 393.

[13] M. F. Shirokov, Gen. Rel. Grav. 4 (1973), 131.

[14] H. Poincaré, Les méthodes nouvelles de la mécanique céleste, Paris, 1892-1899.

[15] Ptolemaios, Almagest; original title ' $H M \alpha \theta \epsilon \mu \alpha \theta \iota \kappa \eta \Sigma v \nu \tau \alpha \xi \iota \varsigma$ (The mathematical syntax), ca. $145 \mathrm{AD}$.

[16] S. Chandrasekhar, The Mathematical Theory of Black Holes, Oxford Univ. Press, New York, 1983.

[17] J. L. Synge, Relativity: the General Theory, Amsterdam, North-Holland, 1960; C. W. Misner, K. S. Thorne and J. A. Wheeler, Gravitation, Freeman, San Francisco, 1970.

[18] S. Weinberg, Gravitation and Cosmology, Academic Press, New York, 1972.

[19] J. Kepler, Astronomia Nova (The New Astronomy), 1609.

[20] L. Landau and E. Lifshitz, Lectures in Theoretical Physics, Vol. 2: Classical Theory of Fields (Pergamon), 1969.

[21] C. G. Darwin, Proc. Roy. Soc. A 249 (1958), 180; Proc. Roy. Soc. A 263 (1961), 39.

[22] N. A. Sharp, Gen. Rel. Grav. 10 (1979), 659.

[23] R. Kerner, J. Martin, S. Mignemi and J. W. van Holten, Phys. Rev. D 63 (2001), 27502.

[24] J. Plebański, Acta Physica Polonica 28 (1965), 141; see also a series of articles by Bażański and Kostyukovich, ibid. B18 (1987), 601, 621, 891, 981.

[25] A. Balakin, J. W. van Holten and R. Kerner, Class. Quantum Grav. 17 (2000), 5009.

[26] R. Kerner, J. W. van Holten and R. Colistete Jr., Relativistic epicycles, preprint grqc/0102099, to appear in Class. Quant. Gravity in 2001.

[27] J. W. van Holten, Fortsch. Phys. 45 (1997), 6.

[28] R. Steinbauer, J. Math. Phys. 39 (1998), 2201. 
[29] R. Kerner, Proceedings of Erevan conference: Classical and Quantum Integrable Systems, JINR: Dubna publications, 1998.

[30] V. Abramov and R. Kerner, J. Math. Phys. 41 (2000), 5998.

[31] S. Kobayashi and K. Nomizu, Foundations of Differential Geometry, Interscience, New York, 1969.

[32] J. L. Synge, Relativity, the General Theory, North-Holland, Amsterdam, 1960.

[33] I. B. Kriphlovich, Sov. Phys. JETP 69 (1989), 217.

[34] A. Barducci, R. Casalbuoni and L. Lusanna, Nuovo Cim. 35A (1976), 377.

[35] L. Brink, P. Di Vecchia and P. Howe, Nucl. Phys. B118 (1977), 76. 